\title{
Clinical Performance of CEA, CA19-9, CA15-3, CA125 and AFP in Gastrointestinal Cancer Using LOCI ${ }^{\mathrm{TM}}$-based Assays
}

\author{
RAMONA C. DOLSCHEID-POMMERICH ${ }^{1}$, STEFFEN MANEKELLER ${ }^{2,3}$, GISELA WALGENBACH-BRÜNAGEL , $^{2,3}$, \\ JÖRG C. KALFF $^{2,3}$, GUNTHER HARTMANN ${ }^{1,3}$, BIRGIT STOFFEL WAGNER ${ }^{1}$ and STEFAN HOLDENRIEDER ${ }^{1,3}$ \\ Departments of ${ }^{1}$ Clinical Chemistry and Clinical Pharmacology, and \\ ${ }^{2}$ Surgery, University Hospital Bonn, Bonn, Germany; \\ ${ }^{3}$ Center for Integrated Oncology (CIO), Köln/Bonn, Germany
}

\begin{abstract}
Background/Aim: Few data are available regarding the clinical performance of LOCI ${ }^{T M}$-based tumor marker assays. We investigated the diagnostic power of carcinogenic antigen, carbohydrate antigen 19-9, carbohydrate antigen 153, carbohydrate antigen 125 and alpha-fetoprotein for detection of gastrointestinal (GI) cancer. Patients and Methods: We analyzed sera from 204 patients (107 with GI cancer, 73 with benign GI diseases and 24 healthy controls) using the Dimension $^{T M}$ Vista1500 analyzer. Results: Levels of biomarkers in healthy controls were in the expected ranges and were only slightly higher in benign GI controls. Established tumor-typeassociated markers were elevated in specific cancer types and discriminated well between cancer and benign controls. Best performance was found for CEA in colorectal cancer (area under the curve $=0.84$, sensitivity $=51.7 \%$ at $95 \%$ specificity $v$ s. benign), CA19-9 in gallbladder/pancreatic cancer $(A U C=0.85$, sensitivity $=60.6 \%)$ and $A F P$ in liver cancer (AUC=0.87, sensitivity=68.4\%). Conclusion: Our study demonstrated the high diagnostic power of well-known biomarkers. LOCI ${ }^{T M}$ based tumor marker assays give reliable results in routine diagnostics.
\end{abstract}

In 2012, there were 14 million new cases of cancer estimated worldwide, with over 8 million cancer-related deaths (1). Among newly-diagnosed solid tumor diseases, a large number were gastrointestinal cancer. Of these, in descending order of their worldwide incidence, 1.36 million were of colorectal cancer followed by 952,000 with gastric cancer, 782,000 with liver cancer was and 456,O00 with esophageal

Correspondence to: Stefan Holdenrieder, MD, Department of Clinical Chemistry and Clinical Pharmacology, Sigmund- FreudStr. 25, D 53127 Bonn, Germany. Tel: +49 22828712126, Fax: +49 22828712159, e-mail: stefan.holdenrieder@uni-bonn.de

Key Words: Gastrointestinal cancer, tumor marker, LOCI $^{\mathrm{TM}}$-based assay, CEA, CA19-9, AFP. cancer, for pancreatic cancer the estimated incidence was 338,000 (2). Despite improvements in the diagnosis and therapy of gastrointestinal cancer, the death rates remain high (3). The worldwide death rates were in descending order 746,000 for liver cancer, 723,000 for gastric cancer, 694,000 for colorectal cancer, 400,000 for oesophageal cancer and 330,000 for pancreatic cancer (2).

Determination of certain tumor marker levels in serum is of fundamental importance e.g. in screening, diagnosis, therapy control and monitoring advanced disease in gastrointestinal cancer (4). In colorectal cancer, carcinogenic antigen (CEA) has a prognostic relevance, with higher CEA levels seeming to be associated with a poorer outcome in a preoperative setting (5). Furthermore CEA is of diagnostic power in postoperative follow-up and early detection of recurrent disease (4). For screening purposes, CEA cannot be used due to its low sensitivity in early cancer stages and the low frequency of cancer in asymptomatic populations (6). Although CEA is an important tumor marker in colorectal cancer, its serum levels can also be elevated in benign diseases (7). In liver cancer, alpha-fetoprotein (AFP) can be used as a screening marker in patients at high risk of developing hepatocellular carcinoma, preoperatively for prognosis evaluation, for postoperative monitoring and also as a tumor marker for monitoring advanced disease. It is of importance that AFP can be elevated not only in hepatocellular carcinoma, but also in other benign diseases and malignancies (8). In gastric cancer, no tumor marker is recommended for screening or diagnosis. (8). In pancreatic cancer, CA19-9 is a well-established tumor marker $(9,10)$ and remains the golden standard tumor marker in follow-up after surgery. The role of CA19-9 in the diagnosis of pancreatic cancer remains unclear (11). CA19-9 is often elevated in advanced gastrointestinal malignancies but also in benign gastrointestinal diseases $(12,13)$. It should be mentioned that CA19-9 is not expressed in individuals with Lewis (a-b-) phenotype, hence in these patients other tumor markers are required (14). 
When new methods for the measurement of tumor markers are established, method comparisons and clinical evaluations are recommended by all important guidelines. Even if two tumor marker methods correlate quite well with each other, in single patients or subgroups, quite different values can be observed. In an earlier study, we showed that chemiluminescent assays in comparison with luminescent oxygen channelling immunoassay ( $\mathrm{LOCI}^{\mathrm{TM}}$ )-based tumor marker assays showed good general correlation for the tumor markers AFP, CEA, CA15-3, prostate-specific antigen (PSA), CA19-9 and carbohydrate antigen (CA125). However, for CA15-3 and CA19-9 greater variations were detected (15, 16). Thus, further studies are needed to evaluate the clinical performance of these tumor marker assays. In order to address important clinical questions patients with cancer have to be compared not only with healthy controls but also with patients with benign diseases. While there are many published studies dealing with the evaluation of tumor markers in gastrointestinal diseases, little research has been done regarding the clinical performance of $\mathrm{LOCI}^{\mathrm{TM}}$-based tumor marker assays in gastrointestinal cancer. LOCI $^{\mathrm{TM}}$-based tumor marker assays are homogenous sandwich chemiluminescent immunoassays which are considered somewhat less specific due to their skipping a washing step. The question, therefore, arises whether these homogenous assays are as good as the established heterogenous assays.

The aim of the present study was to fill this gap by investigating the diagnostic power of $\mathrm{LOCI}^{\mathrm{TM}}$-based tumor marker assays of CEA, CA19-9, CA15-3, CA125 and AFP in patients with gastrointestinal cancer in comparison with healthy controls and patients with benign gastrointestinal diseases.

\section{Patients and Methods}

Patients. In total, 204 patients were included in the present study. Among them were 24 healthy controls, 73 patients with benign gastrointestinal diseases (e.g. diverticulitis, Crohn's disease, colitis, rectal adenoma, gastritis, diaphragmatic hernia, cholecystitis and pancreatitis) and 107 patients with gastrointestinal cancer (35 colorectal cancer, 33 pancreatic/gallbladder cancer, 19 liver cancer and 20 esophageal/gastric cancer). The male to female ratio, median age and range of all included patients are shown in Table I.

All venous punctures for serum collection were at active disease prior to any therapy. All patients and healthy individuals gave their informed consent to blood collection. Samples were collected at the Biofluid Biobank of the University Hospital Bonn at the Institute for Clinical Chemistry and Clinical Pharmacology. The research related to human use complied with all the relevant national regulations, institutional policies and in accordance the tenets of the Helsinki Declaration, and was approved by the local Ethics Committee (approval number: 319-12).

The process of blood handling included immediately delivery to the Central Laboratory of the University Hospital Bonn followed by centrifugation $(3,300 \times g)$ for $10 \mathrm{~min}$. Serum samples were initially stored at $4^{\circ} \mathrm{C}$. Afterwards they were aliquoted into polypropylene vials and stored at $-80^{\circ} \mathrm{C}$. All samples were double-anonomized.
Table I. Patient characteristics. Absolute patient numbers, male to female ratio, median age and range for healthy controls, and individuals with benign gastrointestinal (GI) diseases, liver cancer, gallbladder/pancreatic cancer, colorectal cancer and esophageal/gastric cancer are included.

\begin{tabular}{lccc}
\hline & $\mathrm{N}$ & $\begin{array}{c}\text { Male/female, } \\
\mathrm{n}\end{array}$ & $\begin{array}{c}\text { Median age } \\
\text { (range), years }\end{array}$ \\
\hline Healthy & 24 & $15 / 9$ & $32.4(20.7-78.1)$ \\
Benign GI disease & 73 & $35 / 38$ & $55.2(20.7-91.1)$ \\
Malignant disease & 107 & $71 / 36$ & $68.0(19.6-86.3)$ \\
Liver cancer & 19 & $17 / 2$ & $70.8(57.4-81.4)$ \\
Gallbladder/pancreatic cancer & 33 & $17 / 16$ & $66.5(38.9-79.2)$ \\
Colorectal cancer & 35 & $22 / 13$ & $69.4(19.6-86.3)$ \\
Esophageal/gastric cancer & 20 & $15 / 5$ & $58.1(49.1-82.4)$ \\
\hline
\end{tabular}

Methods. Blood samples were collected and gastrointestinal tumor markers were quantified. The determination of CEA, CA19-9, CA153, CA125 and AFP was performed by LOCI $^{\mathrm{TM}}$-based tumor marker assays for Dimension ${ }^{\text {TM }}$ Vista 1500 analyzer (Siemens Healthcare Diagnostics, Eschborn, Germany). LOCI ${ }^{\mathrm{TM}}$ technology works with a sandwich chemiluminescent immunoassay. Chemibeads contain a chemiluminescent dye and sensibeads contain a photosensitizer dye. Via biotinylated antibodies, both antibody and chemibeads generate sandwiches. Immunocomplexes are formed with further addition of sensibeads. At $680 \mathrm{~nm}$, a chemiluminescent reaction is triggered and ultimately the final signal is detected at $612 \mathrm{~nm}$ (according to the manufacturer's instructions). The tumor marker assays for CEA, CA19-9, CA15-3, CA125 and AFP for the Dimension ${ }^{\mathrm{TM}}$ Vista 1500 analyzer were part of the routine methods of the Central Laboratory of the University Clinics Bonn. Hence internal and external quality control accuracy specifications were fulfilled according to the guidelines of the German Federal Medical Society (RiliB̈̈K) (17). The limit of detection (LoD) and limit of blank (LoB) were as follows: CEA: 0.2 and $0.12 \mathrm{ng} / \mathrm{ml}, \mathrm{CA} 19-9$ : 2.0 and $1.0 \mathrm{U} / \mathrm{ml}$, CA15-3: 1.0 and $0.3 \mathrm{U} / \mathrm{ml}, \mathrm{CA} 125: 1.5$ and $0.5 \mathrm{U} / \mathrm{ml}$ and AFP: 0.5 and $0.2 \mathrm{ng} / \mathrm{ml}$, respectively.

Statistical analysis. Data were statistically analyzed using IBM SPSS Statistics, Version 20 (IBM Corp., Armonk, NY, USA). A value of $p<0.05$ was considered statistically significant. Median, range, and 95th percentile were calculated for all groups. Differences between the collectives were calculated by using Wilcoxon tests. Boxplots depict the value distributions of the different tumor markers. Receiver operating characteristics (ROC) curves show the diagnostic power of CEA, CA19-9, CA15-3, CA125 and AFP. The area under the ROC curves (AUC) and sensitivities at $95 \%$ specificity were computed versus healthy controls and versus benign patients with gastrointestinal diseases.

\section{Results}

The descriptive data for healthy individuals and patients with benign diseases and those in the various subgroups of gastrointestinal cancer are shown in Table II. In healthy individuals AFP, CA125, CA15-3, CA19-9 and CEA values were within the expected range while in those with benign 
Table II. Median, range, and 95th percentiles (95pc) are shown for serum levels of alpha-fetoprotein (AFP), carbohydrate antigen 12-5 (CA125), carbohydrate antigen 15-3 (CA15-3), carbohydrate antigen 19-9 (CA19-9) and carcinoembryonic antigen (CEA) in healthy controls, patients with benign gastrointestinal disease, with liver cancer, gallbladder/pancreatic cancer, colorectal cancer and esophageal/gastric cancer.

\begin{tabular}{|c|c|c|c|c|c|c|}
\hline Group & $\mathrm{N}$ & $\begin{array}{l}\text { AFP }(\mathrm{ng} / \mathrm{ml}) \\
\text { Median } \\
\text { (range; 95pc) }\end{array}$ & $\begin{array}{l}\text { CA125 }(\mathrm{U} / \mathrm{ml}) \\
\text { Median } \\
\text { (range; } 95 \mathrm{pc})\end{array}$ & $\begin{array}{c}\text { CA15-3 (U/ml) } \\
\text { Median } \\
\text { (range; 95pc) }\end{array}$ & $\begin{array}{c}\text { CA19-9 }(\mathrm{U} / \mathrm{ml}) \\
\text { Median } \\
\text { (range; } 95 \mathrm{pc})\end{array}$ & $\begin{array}{l}\text { CEA (ng/ml) } \\
\text { Median } \\
\text { (range; } 95 \mathrm{pc})\end{array}$ \\
\hline Healthy & 24 & $2.5(0.7-7.8 ; 5.8)$ & $8.7(1.8-20.8 ; 14.6)$ & $11.6(5.5-29.7 ; 22.6)$ & $3.2(2.0-17.7 ; 10.1)$ & $1.0(0.5-22.8 ; 2.4)$ \\
\hline Benign & 73 & $2.6(0.8-113.3 ; 9.1)$ & $10.7(2.0-552.9 ; 147.0)$ & $15.5(4.7-54.9 ; 34.9)$ & $6.1(2.0-100.000 ; 86.3)$ & $1.5(0.3-8.6 ; 5.8)$ \\
\hline Liver cancer & 19 & $41.4(0.5-25634 ; 16718)$ & $19.3(5.6-420.9 ; 98.1)$ & $20.0(2.3-110.9 ; 41.1)$ & $17.7(2.0-268.7 ; 171.8)$ & $2.1(0.6-17.5 ; 9.0)$ \\
\hline Colorectal cancer & 35 & $3.2(0.9-26.8 ; 8.8)$ & $11.6(2.7-343.9 ; 108.0)$ & $16.5(6.1-77.9 ; 35.7)$ & $15.2(2.0-100.000 ; 73303) 1$ & $17.1(0.8-45458 ; 17989)$ \\
\hline $\begin{array}{l}\text { Esophageal/ } \\
\text { gastric cancer }\end{array}$ & 20 & $2.6(1.2-80.8 ; 11.6)$ & $11.6(2.2-625.0 ; 81.4)$ & $10.6(3.2-31.0 ; 30.1)$ & $8.8(2.2-429.5 ; 49.6)$ & $2.0(0.7-16.8 ; 6.0)$ \\
\hline $\begin{array}{l}\text { Gallbladder/ } \\
\text { pancreatic cancer }\end{array}$ & 33 & $3.2(1.0-4692.5 ; 211.5)$ & $13.7(5.3-184.3 ; 133.3)$ & $16.5(4.2-84.3 ; 44.6)$ & $98.0(2.0-100.000 ; 15494)$ & $2.1(0.5-38.9 ; 15.4)$ \\
\hline
\end{tabular}

gastrointestinal diseases, slightly higher AFP, CA125, CA153, CA19-9 and CEA values were obtained (see Figure 1). Individual patients with strongly elevated tumor marker levels of CA125 and CA19-9 suffered from hepatitis C with liver cirrhosis.

Liver cancer. Compared to healthy controls, patients with liver cancer had significantly higher levels particularly of AFP $(p<0.001)$, but also of CA19-9 $(p<0.001)$, CEA $(p=0.002)$, CA125 $(p=0.004)$ and CA15-3 $(p=0.044)$. In addition, those with liver cancer compared to those with benign gastrointestinal diseases had significantly higher levels of AFP $(p<0.001)$ and CA19-9 $(p=0.025)$ (Figure 1).

Concerning discrimination of patients with liver cancer from control groups, the best diagnostic performance was achieved for $\mathrm{AFP}$ ( $v s$. healthy controls: $\mathrm{AUC}=0.88$, with $73.7 \%$ sensitivity at $95 \%$ specificity; vs. benign gastrointestinal diseases: $\mathrm{AUC}=0.87$, with $68.4 \%$ sensitivity at $95 \%$ specificity) (Figure 2).

Gallbladder/pancreatic cancer. Compared to healthy controls, patients with gallbladder/pancreatic cancer had significantly higher levels of CA19-9 $(p<0.001)$, but also of CEA $(p \leq 0.001)$, CA125 $(p<0.001)$ and CA15-3 $(p=0.018)$. In addition, levels of CA19-9 $(p<0.001)$ and CEA $(p=0.012)$ were significantly higher in those with gallbladder/pancreatic cancer than in those with benign gastrointestinal diseases (Figure 1).

Regarding discrimination of patients with gallbladder/ pancreatic cancer from control groups, the best diagnostic performance was achieved for CA19-9 ( $v s$. healthy controls: AUC $=0.93$, with $81.8 \%$ sensitivity at $95 \%$ specificity; $v s$. benign gastrointestinal diseases: $\mathrm{AUC}=0.85$, with $60.6 \%$ sensitivity at $95 \%$ specificity) (Figure 2).

Colorectal cancer. Compared to healthy controls, patients with colorectal cancer had particularly significantly higher levels of CEA $(p<0.001)$ and CA19-9 $(p<0.001)$. Values for AFP, CA125 and CA15-3 were not significantly higher. Compared with those with benign gastrointestinal diseases, those with colorectal cancer only had significantly higher levels of CEA $(p<0.001)$ and CA19-9 $(p=0.004)$ (Figure 1).

Concerning discrimination of patients with colorectal cancer from control groups, the best diagnostic performance was achieved for CEA ( $v s$. healthy controls: $\mathrm{AUC}=0.89$, with $80.00 \%$ sensitivity at $95 \%$ specificity; $v s$. benign gastrointestinal diseases: $\mathrm{AUC}=0.84$, with $51.7 \%$ sensitivity at $\%$ specificity) (Figure 2 ).

Esophageal/gastric cancer. Compared to healthy controls, patients with esophageal/gastric cancer had significantly higher levels of CEA $(p=0.001)$ and for CA19- $9(p<0.001)$. However there were no significant differences in any of the tumor markers between those with esophageal/gastric cancer and those with benign gastrointestinal diseases (Figure 1).

\section{Discussion}

The usefulness of tumor marker determination in serum in clinical diagnostics has been shown for many different cancer entities $(18,19)$. The determination of tumor-type-associated tumor markers are recommended in the published guidelines $(4,8)$. When methods for the detection of tumor markers are changed, it is of fundamental importance for the clinical evaluation that parallel comparative measurements are performed. Therefore, in the present study, we investigated the diagnostic power of CEA, CA19-9, CA15-3, CA125 and AFP in patients with gastrointestinal cancer in comparison with healthy controls and benign gastrointestinal diseases as a relevant group for differential diagnosis.

In our previous studies, we showed that regression rates between chemiluminescent and $\mathrm{LOCI}^{\mathrm{TM}}$-based tumor marker assays were acceptable but showed minor differences in 

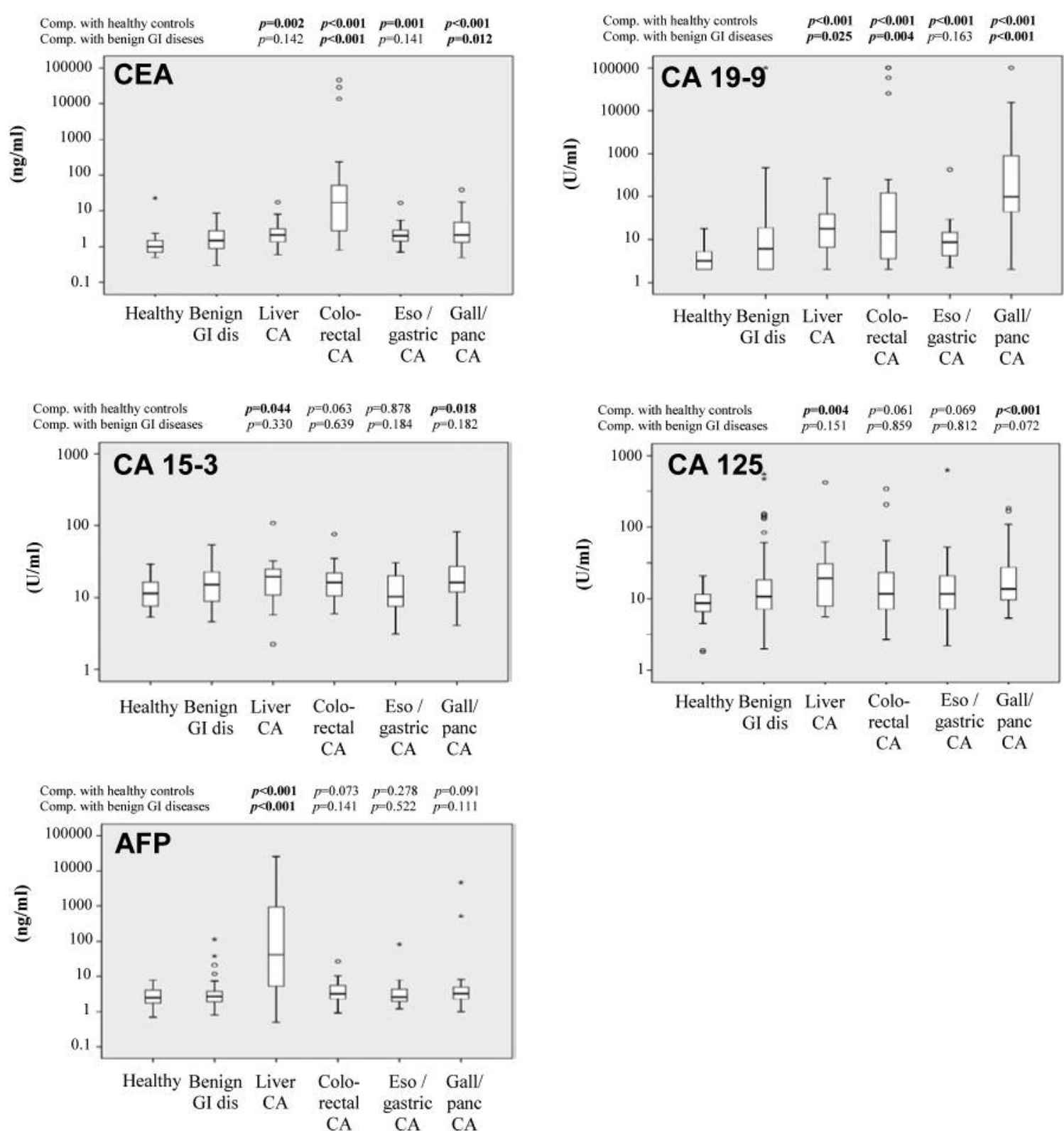

Figure 1. Whisker and boxplots for serum carcinoembryonic antigen (CEA), carbohydrate antigen 19-9 (CA19-9), carbohydrate antigen 15-3 (CA153), carbohydrate antigen 125 (CA125) and alpha-fetoprotein (AFP) values in different diagnostic groups. p-Values were calculated by Wilcoxon tests. Bars represent the median, boxplots shows inter quartile range and whiskers show extreme upper and lower values. Comparison (Comp) are shown for discrimination from other groups. GI: Gastrointestinal, CA: cancer, Dis: diseases, Eso: esophageal, Gall: gallbladder.

subgroups of patients e.g. for AFP $(15,16)$. A major difference in the procedure of homogenous $\mathrm{LOCI}^{\mathrm{TM}}$-assays include the fact that one washing step is left out, which could affect specificity in lower ranges. Regarding the performance of LOCI $^{\mathrm{TM}}$-based assays, Peake et al. showed that for N-terminal pro-B-type natriuretic protein, no significant differences between serum concentrations were found when comparing the Dimension Vista LOCI ${ }^{\mathrm{TM}}$ method with the Siemens Immulite
2500 (20). For cardiac troponin I, an acceptable analytical performance of the $\mathrm{LOCI}^{\mathrm{TM}}$ method on the Dimension EXL (Siemens Healthcare Diagnostics) was found (21). The evaluation of the analytical performance of CA19-9 using non LOCI $^{\mathrm{TM}}$-based immunoassays revealed minimal differences in slopes and intercepts when comparing two different immunoassays; good correlation was shown for all patients in their collective (22). In addition, for CA15-3 the analytical 
Liver CA vs. Healthy

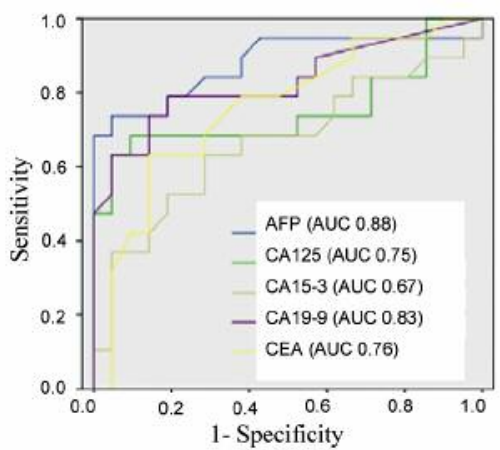

Gall Bladder/Panc CA vs. Healthy

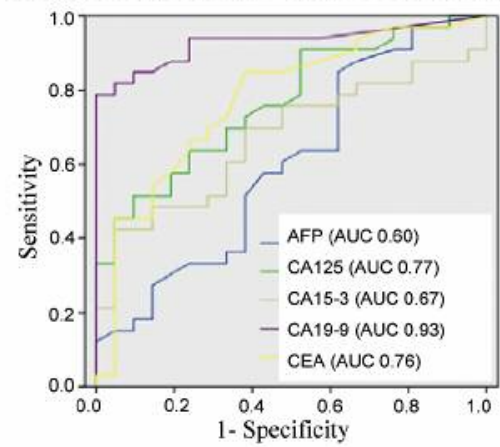

Colorectal CA vs. Healthy

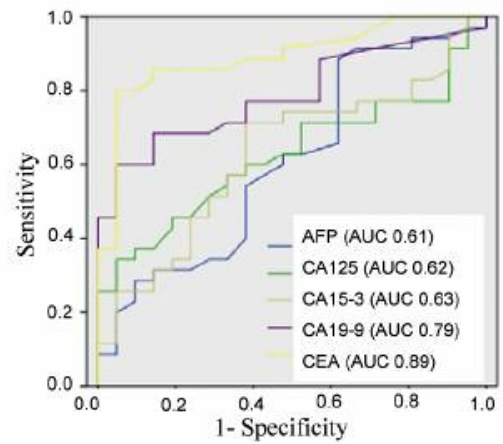

Liver CA vs. Benign

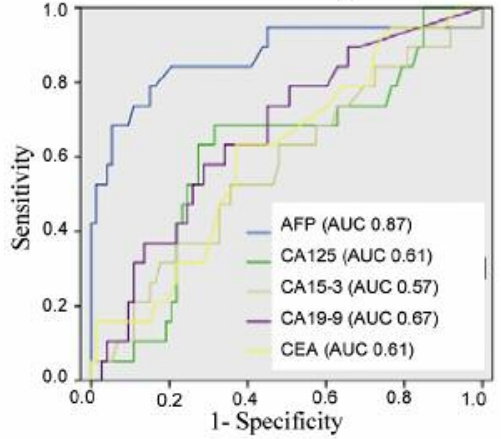

Gall Bladder / Panc CA vs. Benign

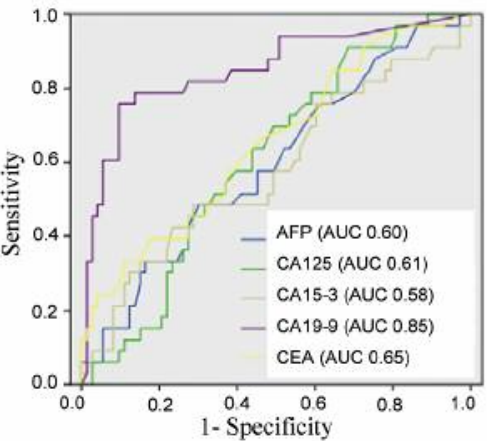

Colorectal CA vs. Benign

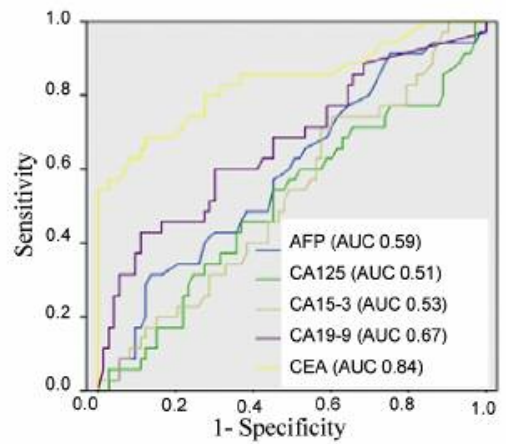

Esophageal/ Gastric CA vs. Healthy Esophageal/ Gastric CA vs. Benign
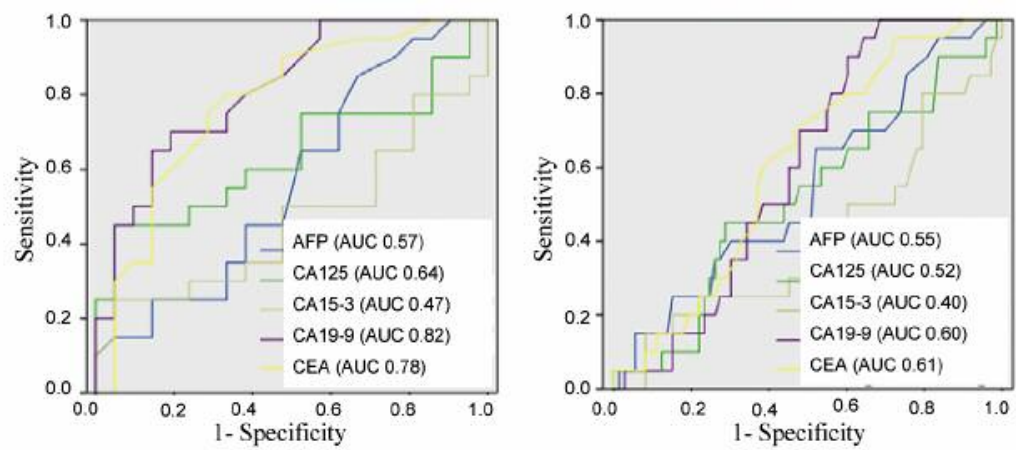

Figure 2. Receiver operating characteristic curves and areas under the curve (AUC) for the discrimination between patients with gastrointestinal tumors versus healthy controls and versus those with benign gastrointestinal diseases using serum alphafetoprotein (AFP), carbohydrate antigen 125 (CA125), carbohydrate antigen 15-3 (CA15-3), carbohydrate antigen 19-9 (CA19-9) and carcinoembryonic antigen (CEA) in liver cancer, gallbladder/ pancreatic cancer, colorectal cancer, oesophageal/gastric cancer. 
performance was accurate but not optimal and a difference in slope of 20-40\% lower concentrations for the applied method was detected (23). Looking at the analytical performance of CA125, excellent correlation and only minimal differences in slopes and intercepts were reported (24). We conclude that analytical challenges and limitations are seen for most different methods applied in the routine measurement of tumor markers. In order to reach a high diagnostic power, our study not only compared healthy controls and patients with tumors, but also included patients with benign gastrointestinal diseases, which are of immense discriminative diagnostic importance $(25,26)$. We showed that the LOCI $^{\mathrm{TM}}$-based tumor marker assays detected our healthy control group correctly. These results were in line with healthy control data presented by the manufacturer. In accordance with previous findings, our study further showed that many markers discriminated well between healthy controls and patients with gastrointestinal cancer, but only tumor-typeassociated markers discriminated between benign controls and those with cancer $(4,27)$.

CA19-9 has a sensitivity of $70-80 \%$ in pancreatic cancer (independently of tumor differentiation) (28). We showed that in pancreatic and gallbladder cancer, best diagnostic power was achieved by CA19-9, with an AUC of 0.85 and a sensitivity of $60.6 \%$ at a $95 \%$ specificity versus benign gastrointestinal diseases. These results are in line with earlier study on pancreatic cancer that described AUCs of 0.827 (sensitivity of $53.2 \%$ at $95 \%$ specificity) and 0.850 (sensitivity of $58.1 \%$ at $95 \%$ specificity) when comparing them with benign gastrointestinal diseases using the Access ${ }^{\mathrm{TM}}$ GI Monitor and the Elecsys $^{\text {TM }}$ CA19-9 (22). In colorectal cancer, best performance was achieved by CEA with an AUC of 0.84 and a sensitivity of $51.7 \%$ at $95 \%$ specificity versus benign gastrointestinal diseases. Another study reported an AUC of 0.79 and a sensitivity of $64.5 \%$ of CEA when comparing colorectal cancer with benign colorectal diseases (29). In liver cancer, we showed that AFP with an AUC of 0.87 and a sensitivity of $68.4 \%$ at $95 \%$ specificity versus benign gastrointestinal diseases performed best. AFP has a high specificity for a hepatocellular carcinoma. Particularly in patients with benign liver diseases such as liver cirrhosis and chronic hepatitis, AFP is used as a screening tool for hepatocellular carcinoma (28). Therefore, it is of crucial importance to look at the discriminative power of the assay used in comparison with benign diseases. In esophageal and gastric cancer, no marker was found to be of high diagnostic power. This was in line with other studies (2224). Single elevated tumor markers were measured in patients suffering from hepatitis $\mathrm{C}$ with liver cirrhosis. Earlier findings discuss elevated levels of e.g. CA125 and CA19-9 due to effusion or cholestasis $(30,31)$.

In the present study, we respected the requirements for clinical tumor marker evaluation as suggested by the European Group on Tumor Markers by including samples from patients with cancer, healthy controls and benign organ-related diseases as relevant groups for differential diagnosis. To achieve real-time diagnostic settings, samples were measured under routine conditions including pre-analytic handling and internal and external quality controls. However, samples were collected in a standardized manner at the University Biofluid Biobank and analyzed in batches for study purposes.

While the number of samples in the present study is limited, we assessed all available LOCI ${ }^{\mathrm{TM}}$-based markers in our setting and confirmed earlier findings derived with other methods.

In our study, we evaluated the clinical performance of LOCI $^{\text {TM }}$-based tumor marker assays CEA, CA19-9, CA15-3, CA125 and AFP in patients with gastrointestinal cancer and demonstrated their high diagnostic power. LOCI ${ }^{\mathrm{TM}}$-based tumor marker assays give reliable results in routine diagnostics.

\section{Acknowledgements}

None.

\section{References}

1 Ferlay J, Soerjomataram I, Dikshit R, Eser S, Mathers C, Rebelo M, Parkin DM, Forman D and Bray F: Cancer incidence and mortality worldwide: sources, methods and major patterns in GLOBOCAN 2012. Int J Cancer 136: 359-386, 2015.

2 Stewart BW and Wild CP: World Cancer Report 2014 Lyon: International Agency for Research on Cancer; Lyon, France, 2014.

3 Siegel RL, Miller KD and Jemal A: Cancer statistics, 2015. CA Cancer J Clin 65: 5-29, 2015.

4 Sturgeon CM, Duffy MJ, Stenman UH, Lilja H, Brünner N, Chan DW, Babaian R, Bast RC Jr, Dowell B, Esteva FJ, Haglund C, Harbeck N, Hayes DF, Holten-Andersen M, Klee GG, Lamerz R, Looijenga LH, Molina R, Nielsen HJ, Rittenhouse H, Semjonow A, Shih IeM, Sibley P, Sölétormos G, Stephan C, Sokoll L, Hoffman BR, Diamandis EP; National Academy of Clinical Biochemistry: National Academy of Clinical Biochemistry laboratory medicine practice guidelines for use of tumor markers in testicular, prostate, colorectal, breast, and ovarian cancers. Clin Chem 54: 11-79, 2008.

5 Thirunavukarasu P, Sukumar S, Sathaiah M, Mahan M, Pragatheeshwar KD, Pingpank JF, Zeh H 3rd, Bartels CJ, Lee KK and Bartlett DL: C-stage in colon cancer: implications of carcinoembryonic antigen biomarker in staging, prognosis, and management. J Natl Cancer Inst 103: 689-697, 2011.

6 Thomas DS, Fourkala EO, Apostolidou S, Gunu R, Ryan A, Jacobs I, Menon U, Alderton W, Gentry-Maharaj A and Timms JF: Evaluation of serum CEA, CYFRA21-1 and CA125 for the early detection of colorectal cancer using longitudinal preclinical samples. Br J Cancer 113: 268-274, 2015.

7 Ruibal Morell A: CEA serum levels in non-neoplastic disease. Int J Biol Markers 7: 160-166, 1992.

8 Sturgeon CM, Duffy MJ, Hofmann BR, Lamerz R, Fritsche HA, Gaarenstroom K, Bonfrer J, Ecke TH, Grossman HB, Hayes P, Hoffmann RT, Lerner SP, Löhe F, Louhimo J, Sawczuk I, Taketa K, Diamandis EP; National Academy of Clinical Biochemistry: National Academy of Clinical Biochemistry Laboratory Medicine Practice Guidelines for use of tumor markers in liver, bladder, cervical, and gastric cancers. Clin Chem 56: 1-48, 2010. 
9 Ferrone CR, Finkelstein DM, Thayer SP, Muzikansky A Fernandez-del Castillo C and Warshaw AL: Perioperative CA199 levels can predict stage and survival in patients with resectable pancreatic adenocarcinoma. J Clin Oncol 24: 2897-2902, 2006.

10 Berger AC, Garcia M Jr, Hoffman JP, Regine WF, Abrams RA, Safran H, Konski A, Benson AB 3rd, MacDonald J and Willett CG: Postresection CA19-9 predicts overall survival in patients with pancreatic cancer treated with adjuvant chemoradiation: a prospective validation by RTOG 9704. J Clin Oncol 26: 59185922, 2008.

11 Duffy MJ, Sturgeon C, Lamerz R, Haglund C, Holubec VL, Klapdor R, Nicolini A, Topolcan O and Heinemann V: Tumor markers in pancreatic cancer: a European Group on Tumor Markers (EGTM) status report. Ann Oncol 21: 441-447, 2010.

12 Steinberg W: The clinical utility of the CA19-9 tumor-associated antigen. Am J Gastroenterol 85: 350-355, 1990.

13 Duffy MJ: CA19-9 as a marker for gastrointestinal cancers: a review. Ann Clin Biochem 35: 364-370, 1998.

14 Tempero MA, Uchida E, Takasaki H, Burnett DA, Steplewski Z and Pour PM: Relationship of carbohydrate antigen 19-9 and Lewis antigens in pancreatic cancer. Cancer Res 47: 5501-5503, 1987.

15 Zur B, Holdenrieder S, Albers E, Walgenbach-Brünagel G and Stoffel-Wagner B: Method comparison for CA15-3, CA19-9, and CA125 determination using the new LOCI technique of Dimension Vista 1500 and Immulite 2000 XPI. J Immunoassay Immunochem 33: 435-445, 2012.

16 Zur B, Holdenrieder S, Walgenbach-Brünagel G, Albers E and Stoffel-Wagner B: Method comparison for determination of the tumor markers AFP, CEA, PSA and free PSA between Immulite 2000 XPI and Dimension Vista 1500. Clin Lab 58: 97-105, 2012.

17 Richtlinie der Bundesärztekammer zur Qualitätssicherung laboratoriumsmedizinischer Untersuchungen - Rili-BÄK: Deutsches Ärzteblatt 38, Germany 2014.

18 Huh JW, Oh BR, Kim HR and Kim YJ: Preoperative carcinoembryonic antigen level as an independent prognostic factor in potentially curative colon cancer. J Surg Oncol 101: 396-400, 2010.

19 Haas M, Heinemann V, Kullmann F, Laubender RP, Klose C, Bruns CJ, Holdenrieder S, Modest DP, Schulz C and Boeck S: Prognostic value of CA19-9, CEA, CRP, LDH and bilirubin levels in locally advanced and metastatic pancreatic cancer: results from a multicenter, pooled analysis of patients receiving palliative chemotherapy. J Cancer Res Clin Oncol 139: 681-689, 2013.

20 Peake RW, Turner HE, Leaper W , Deans KA, Hannah A and Croal BL: Comparison of sample types for N-terminal pro-Btype natriuretic peptide measured on the Siemens Immulite 2500 and Dimension Vista LOCI methods. Ann Clin Biochem 49: 494-496, 2012.

21 Calderón JLM, Varona Pérez JM, Bustos F and Sánchez Gómez JC: Performance characteristics of loci method for measuring cardiac troponin I on the dimension EXL. Practical Laboratory Medicine 1: 42-47, 2015.
22 Stieber P, Molina R, Gion M, Gressner A, Troalen F, Holdenrieder S, Auge JM, Zancan M, Wycislo M and Jarrige V: Alternative antibody for the detection of CA19-9 antigen: a European multicenter study for the evaluation of the analytical and clinical performance of the Access GI Monitor assay on the UniCel Dx1 800 Immunoassay System. Clin Chem Lab Med 46: 600-611, 2008.

23 Molina R, Gion M, Gressner A, Troalen F, Auge JM, Holdenrieder $\mathrm{S}$, Zancan $\mathrm{M}$, Wycislo $\mathrm{M}$ and Stieber $\mathrm{P}$ : Alternative antibody for the detection of CA15-3 antigen: a European multicenter study for the evaluation of the analytical and clinical performance of the Access BR Monitor assay on the UniCel Dxl 800 Immunoassay System. Clin Chem Lab Med 46: 612-622, 2008

24 Holdenrieder S, Molina R, Gion M, Gressner A, Troalen F, Auge JM, Zancan M, Wycislo M and Stieber P: Alternative antibody for the detection of CA125 antigen: a European multicenter study for the evaluation of the analytical and clinical performance of the Access OV Monitor assay on the UniCel Dxl 800 Immunoassay System. Clin Chem Lab Med 46: 588-599, 2008.

25 Dranka-Bojarowska D, Lekstan A, Olakowski M, Jablonska B, Lewinski A, Musialski P, Sobczyk W, Kapalka A and Lampe P: The assessment of serum concentration of adiponectin, leptin and serum carbohydrate antigen-19.9 in patients with pancreatic cancer and chronic pancreatitis. J Physiol Pharmacol 66: 653663, 2015.

26 Scarà S, Bottoni P and Scatena R: CA19-9: Biochemical and Clinical Aspects. Adv Exp Med Biol 867: 247-260, 2015.

27 Duffy MJ: Tumor markers in clinical practice: a review focusing on common solid cancers. Med Princ Pract 22: 4-11, 2013.

28 Stieber P and Heinemann V: Sinnvoller Einsatz von Tumormarkern. J Lab Med 32: 339-360, 2008.

29 Zhang SY, Lin $M$ and Zhang HB: Diagnostic value of carcinoembryonic antigen and carcinoma antigen 19-9 for colorectal carcinoma. Int J Clin Exp Pathol 8: 9404-9409, 2015.

30 Trapé J, Filella X, Alsina-Donadeu M , Juan-Pereira L, BoschFerrer Á, Rigo-Bonnin R; Oncology Section of the Catalan Association of Clinical Laboratory Science: Increased plasma concentrations of tumour markers in the absence of neoplasia. Clin Chem Lab Med 49: 1605-1620, 2011.

31 Trapé J, Gurt G, Franquesa J, Montesinos J, Arnau A, Sala M, Sant F, Casado E, Ordeig JM, Bergos C, Vida F, Sort P, Isava Á, González $\mathrm{M}$ and Molina R: Diagnostic Accuracy of Tumor Markers CYFRA21-1 and CA125 in the Differential Diagnosis of Ascites. Anticancer Res 35: 5655-5660, 2015.

Received November 15, 2016

Revised December 9, 2016

Accepted December 16, 2016 